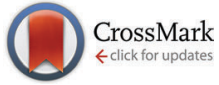

Cite this: J. Mater. Chem. C, 2016, 4, 1813

Received 13th January 2016 , Accepted 1st February 2016

DOI: 10.1039/c6tc00170j

www.rsc.org/MaterialsC

\title{
Thermodynamic stability and control of oxygen reactivity at functional oxide interfaces: EuO on ITO†
}

\author{
Timm Gerber, ${ }^{\text {a }}$ Patrick Lömker, ${ }^{a}$ Bernardus Zijlstra, ${ }^{a}$ Claire Besson, $\ddagger^{b}$ \\ David N. Mueller, ${ }^{a}$ Willi Zander, ${ }^{c}$ Jürgen Schubert, ${ }^{c}$ Mihaela Gorgoi ${ }^{d}$ and \\ Martina Müller*ae
}

\begin{abstract}
As a prototypical all-oxide heterostructure, the ferromagnetic insulator europium monoxide (EuO) is synthesized on transparent and conductive indium tin oxide (ITO) virtual substrates. Non-destructive hard X-ray photoelectron spectroscopy is employed to depth profile the chemical composition of the magnetic layer and the buried oxide-oxide interface. We find that thermally activated oxygen diffusion from ITO affects the EuO growth process. We present how to control the oxygen reactivity at the interface and discuss its origin in a thermodynamic analysis. Our complementary methodical strategy allows for a significant improvement of the EuO chemical quality with sizeable magnetic properties. Generally, our approach derives guidelines for the proper choice of oxide substrates and buffer layer materials for functional all-oxide heterostructures.
\end{abstract}

\section{Introduction}

Functional metal oxides (MOs) are at the heart of such diverse research areas as nano-, spin- and optoelectronics as well as catalysis, battery- and solid oxide fuel cell technology. An exciting prospect in the field of oxide spintronics is the combination of an insulating magnetic oxide with a conductive oxide electrode. Such a magnetic tunnel junction can be used to generate highly spin-polarized currents. ${ }^{1,2}$ In this work, we propose and study a prototype heterostructure for such application, which is composed of a ferromagnetic insulator, europium monoxide (EuO), and a transparent conducting electrode, Sn-doped $\mathrm{In}_{2} \mathrm{O}_{3}$ (ITO). ${ }^{3}$ In addition to the 'spin filter' ability, the transparency of the substrate allows for in operando probing of the EuO's magneto-optical response. ${ }^{4}$

High-quality MOs are typically synthesized by thoroughly controlled high-temperature metal-oxygen reactions. ${ }^{5}$ Integrating MOs into multifunctional oxide heterostructures requires a

\footnotetext{
${ }^{a}$ Peter Grünberg Institut (PGI-6), Forschungszentrum Jülich, Jülich, Germany.

E-mail: t.gerber@fz-juelich.de, mart.mueller@fz-juelich.de

${ }^{b}$ Institut für Anorganische Chemie, RWTH Aachen University, Germany

${ }^{c}$ Peter Grünberg Institut (PGI-9), Forschungszentrum Jülich, Jülich, Germany

${ }^{d}$ Helmholtz-Zentrum für Materalien und Energie GmbH, Berlin, Germany

${ }^{e}$ Fakultär für Physik, Universität Duisburg-Essen, Duisburg, Germany

$\dagger$ Electronic supplementary information (ESI) available: Additional HAXPES data and details on the fitting procedures. See DOI: 10.1039/c6tc00170j

\# Present address: Department of Chemistry, George Washington University, Washington, District Of Columbia, USA.
}

fundamental understanding of these chemical processes, in order to prepare stable oxide phases with atomically sharp interfaces. The careful control of elemental fluxes and temperature is particularly important for growing metastable ferromagnetic EuO with its very narrow parameter range for stoichiometric synthesis: if the ratio of europium metal and oxygen flux $J_{\mathrm{Eu}} / J_{\mathrm{O}}$ only slightly deviates from unity, either Eu metal-rich $\mathrm{EuO}_{1-x}$ or the overoxidized, paramagnetic phases $\mathrm{Eu}_{3} \mathrm{O}_{4}$ and $\mathrm{Eu}_{2} \mathrm{O}_{3}$ are formed. ${ }^{6}$ By applying an adsorption-controlled EuO growth mode, the so-called distillation method, the narrow parameter space can be expanded. This method implies a flux ratio $J_{\mathrm{Eu}} / J_{\mathrm{O}}>1$ and an elevated growth temperature which is high enough to enable the re-evaporation of excess Eu metal. ${ }^{6-11}$

At elevated growth temperatures, however, chemical reactions between the functional EuO layer and the substrate may occur, thereby yielding undesired interface contaminants. On Si substrates, these processes can be suppressed by the careful choice of buffer layers e.g. $\mathrm{SiO}_{x}{ }^{12}$ or $\mathrm{SrO},{ }^{13}$ as well as by surface passivation e.g. through formation of EuSi. ${ }^{14}$ Thermodynamic stability of EuO with the substrate or buffer layer is important because it signals the absence of a driving force for such reactions. ${ }^{15}$ While an exhaustive knowledge of the thermodynamic stability of semiconductor-oxide ${ }^{16}$ and metal-oxide ${ }^{17,18}$ interfaces is available in literature, fundamental insights into the thermochemistry of all-oxide heterostructures are surprisingly sparse.

In this regard, we investigate the impact of the thermodynamic properties on the growth, interface chemistry and magnetic functionality of EuO on ITO virtual substrates. We employ hard 


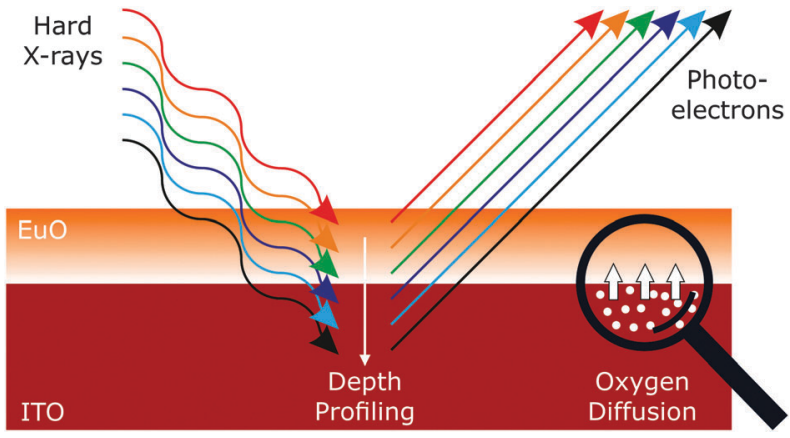

Fig. 1 Principle of a HAXPES depth profile: the escape depth of the photoelectrons depends on the chosen excitation energy.

X-ray photoelectron spectroscopy (HAXPES) as a powerful nondestructive and element-sensitive probing technique. ${ }^{19,20}$ HAXPES enables the chemical depth profiling of the functional oxide layer and the buried heterointerfaces, as schematically depicted in Fig. 1 . We show that thermally activated oxygen diffusion at the $\mathrm{EuO} / \mathrm{ITO}$ interface limits the application of the otherwise beneficial EuO distillation method. Our study is corroborated by a comprehensive thermodynamic analysis of the EuO/ITO heterosystem. Applying these complementary methods yields a fundamental understanding of the origin of the oxygen reactivity at the $\mathrm{EuO} / \mathrm{ITO}$ interface, and allows us to significantly optimize the stability of ferromagnetic EuO on ITO virtual substrates. In view of the significance to control oxygen reactivity at oxide interfaces, we derive guidelines for a suitable choice of substrates and buffer layer materials for all-oxide heterostructures.

\section{Experimental}

Virtual ITO substrates were prepared by depositing $50 \mathrm{~nm}$ thick ITO films via pulsed laser deposition (PLD) onto epipolished (001)-oriented yttria-stabilized zirconia (YSZ, composition $\left.\mathrm{Zr}_{0.87} \mathrm{Y}_{0.13} \mathrm{O}_{1.935}\right)$. ITO/YSZ(001) heteroepitaxy is achievable, because the YSZ lattice constant $a_{\mathrm{YsZ}}=5.142 \AA$ almost equals half that of ITO $\left(a_{\mathrm{ITO}}=10.13 \AA\right)$, with a tensile lattice mismatch of $a_{\mathrm{ITO}} /\left(2 \cdot a_{\mathrm{YSZ}}\right) \approx+1.5 \% .{ }^{21}$ PLD deposition of ITO was performed using a target with the composition $\operatorname{In}_{1.8} \mathrm{Sn}_{0.2} \mathrm{O}_{3}$ (Mateck $\mathrm{GmbH}$, Jülich), a KrF excimer laser (wavelength $248 \mathrm{~nm}, 40 \mathrm{~ns}$ and $1 \mathrm{~J}$ per pulse) with an $\mathrm{O}_{2}$ background pressure $p_{\mathrm{O}_{2}}=5 \times$ $10^{-3}$ mbar. $^{22}$ During ITO growth, the YSZ substrate temperature was set to $T_{\mathrm{S}} \approx 300{ }^{\circ} \mathrm{C}$.

Thereafter, the ITO virtual substrates were transferred $e x$ situ to an MBE system with a residual gas pressure $p<10^{-10}$ mbar, where they were annealed in ultrahigh vacuum at $T_{\mathrm{S}}=550{ }^{\circ} \mathrm{C}$ for $t=2 \mathrm{~h}$. For subsequent EuO growth, the substrate temperature was set to either room temperature (RT) or $400{ }^{\circ} \mathrm{C}$. Stoichiometric EuO was synthesized by e-beam evaporating 99.99\% pure Eu with a deposition rate of $0.13 \AA_{\mathrm{s}}^{-1}$ in an $\mathrm{O}_{2}$ atmosphere. Using specialized gas inlets, the oxygen gas was equally distributed to the sample surface and to a quadrupole

$\S$ We note that ITO is stable under these conditions (see Fig. 6). mass spectrometer (QMS) which was used to carefully control the partial $\mathrm{O}_{2}$ pressure. We applied a flux ratio of $J_{\mathrm{Eu}} / J_{\mathrm{O}}=1$ which in our setup corresponds to $p_{\mathrm{O}_{2}}=1.7 \times 10^{-9} \mathrm{mbar}$ at the QMS. This value was determined by growing EuO on YSZ with varying $\mathrm{O}_{2}$ pressure. ${ }^{23}$ EuO growth was always initiated by exposing the sample to Eu metal flux. Subsequently, the $\mathrm{O}_{2}$ flux was ramped to the desired value within $c a .30$ seconds.

Structural analysis of the EuO/ITO samples was carried out in situ by reflection high-energy electron diffraction (RHEED) and low-energy electron diffraction (LEED). Further ex situ structural analysis was carried out by X-ray diffraction (XRD) using a Philips X'Pert MRD four-circle diffractometer. Prior to all ex situ measurements the samples were capped with an Al layer $(d=10 \mathrm{~nm})$ to prevent further oxidation of the metastable EuO films in air.

Magnetic characterization was carried out using a Quantum Design superconducting quantum interference device (SQUID) magnetometer. In-plane hysteresis loops were measured at $T=5 \mathrm{~K}$ and $M(T)$ curves were recorded warming up the sample in an alignment field of 100 Oe. The data was corrected for a paramagnetic background caused by the substrate and normalized by the nominal thickness of the EuO layer.

For chemical analysis and element-selective depth profiling, hard X-ray photoemission spectroscopy (HAXPES) experiments were conducted at the KMC-1 beamline at BESSY II (Berlin) with the HIKE endstation. ${ }^{24} \mathrm{Si}(111)$ and $\mathrm{Si}(311)$ double crystal monochromators were used to generate photon energies between $2010 \mathrm{eV}$ and $5000 \mathrm{eV}$. The total energy resolution ranges from $320 \mathrm{meV}$ to $500 \mathrm{meV}$ and the information depth from $\sim 10 \mathrm{~nm}$ to $\sim 25 \mathrm{~nm}$. The binding energy scale was calibrated by measuring the $4 \mathrm{f}$ level of an $\mathrm{Au}$ foil attached to the sample manipulator. Eu $3 \mathrm{~d}$ and In $3 \mathrm{~d}$ spectra were recorded at room temperature and in normal emission geometry. For quantitative analysis the spectra were corrected by Tougaard backgrounds to account for inelastic photoelectron scattering and fitted by convoluted Gaussian-Lorenzian peak shapes. The relative error of intensities estimated in this way was assumed to be $10 \%$.

Three types of EuO/ITO samples are discussed in the following: type (i): $4 \mathrm{~nm} \mathrm{EuO/ITO}$ with the EuO layer grown at $T_{\mathrm{S}}=400{ }^{\circ} \mathrm{C}$, type (ii): $4 \mathrm{~nm} \mathrm{EuO/2} \mathrm{nm} \mathrm{YSZ/ITO} \mathrm{with} \mathrm{the} \mathrm{EuO} \mathrm{layer} \mathrm{grown} \mathrm{at}$ $400{ }^{\circ} \mathrm{C}$, but with an additional PLD-grown $2 \mathrm{~nm}$ thick YSZ buffer layer, and type (iii): $4 \mathrm{~nm} \mathrm{EuO/ITO}$ with the EuO layer grown at room temperature (RT).

\section{Results and discussion}

\subsection{ITO virtual substrates}

First, we characterize the ITO/YSZ(001) virtual substrates utilized for subsequent EuO growth. Fig. 2(a) shows the XRD pattern of a $50 \mathrm{~nm}$ thick ITO film on YSZ(001). Only intense peaks of ITO(00l) and $\mathrm{YSZ}(00 l)$ are observed, thereby confirming the epitaxial relation ITO(001)||YSZ(001). A rocking curve of the ITO(006) peak (data not shown) yields a full width at half maximum (FWHM) of only $0.4^{\circ}$, which is comparable with previous work. ${ }^{21}$ From a Nelson-Riley analysis of the $\mathrm{ITO}(00 l)$ peaks we determine 

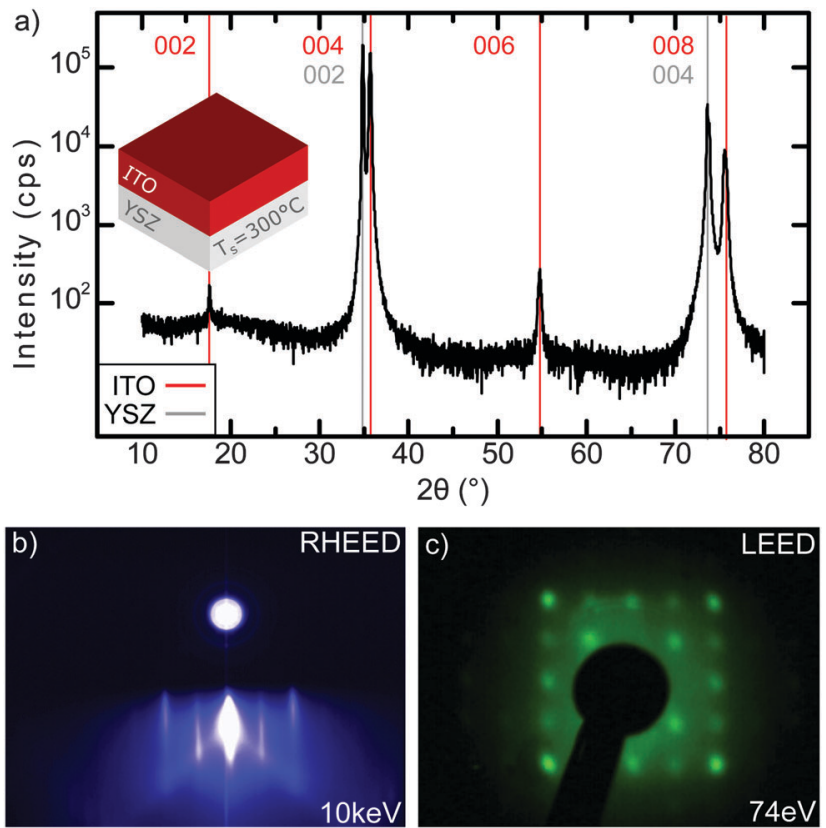

Fig. 2 Structural characterization of a $50 \mathrm{~nm}$ ITO film deposited on YSZ. (a) XRD scan with out-of-plane diffraction peaks of ITO (red) and YSZ (gray), (b) RHEED image taken with $10 \mathrm{keV}$ at a substrate temperature $T_{\mathrm{S}}=400{ }^{\circ} \mathrm{C}$, (c) LEED image recorded with $74 \mathrm{eV}$ at $T_{\mathrm{S}}=225^{\circ} \mathrm{C}$.

an out-of-plane lattice constant $a_{\mathrm{ITO}}=10.106 \AA$, which is $0.21 \%$ smaller than the ITO bulk lattice parameter. This uniaxial compression of ITO is likely due to the counteracting in-plane tensile strain of $+1.5 \%$ induced by the YSZ substrate.

Fig. 2(b) and (c) show RHEED and LEED images of the ITO/ YSZ(001) substrates after annealing in UHV. We observe sharp streaks in the RHEED pattern, which indicate an atomically flat ITO(001) surface. The LEED pattern shows cubic symmetry, whereby the second-order ITO reflections coincide with the first-order reflections of the YSZ substrate. We thus conclude, that the ITO film grows epitaxial on YSZ with the cube-on-cube orientation $\operatorname{ITO}(001)|| Y S Z(001)$ and $\operatorname{ITO}(010)|| Y S Z(010)$, fully consistent with the ex situ XRD data.

\subsection{EuO on ITO: depth-profiling of chemical composition}

Next, the properties of EuO ultrathin films $(d=4 \mathrm{~nm})$ grown on ITO(001) virtual substrates are discussed. For sample type (i), we applied an EuO growth mode with $T_{\mathrm{S}}=400{ }^{\circ} \mathrm{C}$ and a flux ratio $J_{\mathrm{Eu}} / J_{\mathrm{O}}=1$, which yields perfectly stoichiometric and singlecrystalline EuO thin films on cubic oxide substrates e.g. YSZ. ${ }^{23}$ Fig. 3(b) shows the RHEED pattern of sample type (i). We observe diffraction spots characteristic for epitaxial growth. However, the spots are very broad indicating a rough surface and threedimensional island growth.

In order to examine the depth-dependent chemical properties of EuO/ITO sample type (i), we used HAXPES to investigate the Eu 3d core level. This core level is spin-orbit (SO) split into a $3 \mathrm{~d}_{5 / 2}$ and $3 \mathrm{~d}_{3 / 2}$ component separated by $E_{\text {SO }}=30 \mathrm{eV} .^{25}$ The $3 \mathrm{~d}_{5 / 2}$ component is shown in Fig. 3(c). It reveals a chemical shift between divalent $\mathrm{Eu}^{2+}$ contributions - the signature of stoichiometric and ferromagnetic $\mathrm{EuO}$ - and trivalent $\mathrm{Eu}^{3+}$, which is assigned to over-oxidized phases. For a photon energy of $2010 \mathrm{eV}$, the fit yields a spectral weight of the $\mathrm{Eu}^{2+}$ components of $27 \%$ and of $\mathrm{Eu}^{3+}=73 \%$. Obviously, a major amount of over-oxidized phases has formed during growth despite applying a flux ratio of $J_{\mathrm{Eu}} / J_{\mathrm{O}}=1$.

In order to get depth-sensitive information of the chemical composition, we recorded the Eu $3 \mathrm{~d}_{5 / 2}$ multiplet with the incident photon energy varying from $2010 \mathrm{eV}$ to $5000 \mathrm{eV}$, as shown in Fig. 3(c). The corresponding fit results are summarized in Fig. 4(a). We observe, that the relative intensity of the $\mathrm{Eu}^{2+}$ component gradually decreases with increasing photon energy, i.e. with increasing bulk sensitivity. This result indicates, that the amount of stoichiometric EuO decreases from the surface towards the EuO/ITO interface.

We therefore conclude that - although the $\mathrm{O}_{2}$ flux is finetuned to match the Eu flux during growth - the ITO virtual substrate likely acts as an additional source of oxygen, resulting in a significantly enhanced fraction of over-oxidized EuO phases mainly located at the EuO/ITO interface. Consequently, the growth conditions need to be refined for synthesis of stoichiometric EuO on ITO.

In the following, we discuss two strategies to suppress the oxygen diffusion from the ITO virtual substrate during EuO growth.

First, we apply the same growth conditions $\left(T_{\mathrm{S}}=400{ }^{\circ} \mathrm{C}\right.$, $J_{\mathrm{Eu}} / J_{\mathrm{O}}=1$ ) but insert a $2 \mathrm{~nm}$ thick PLD-grown YSZ buffer layer on top of the ITO virtual substrate (sample type (ii)). YSZ was chosen as a buffer layer material, since EuO grows with high crystalline quality directly on YSZ substrates. ${ }^{8}$ The results are compiled in the middle row of Fig. 3. The RHEED pattern in Fig. 3(f) shows diffraction spots which are characteristic for threedimensional island growth. However, they are sharper than those observed for sample type (i), thus suggesting a lower surface roughness of the EuO film grown on $2 \mathrm{~nm}$ YSZ/ITO. From the HAXPES depth profile in Fig. $3(\mathrm{~g})$, we find that the spectral intensity of the $\mathrm{Eu}^{2+}$ components is $60 \%$ for the lowest photon energy (i.e. largest surface sensitivity) and decreases gradually to $25 \%$ for the largest photon energy of $5000 \mathrm{eV}$ (i.e. largest bulk sensitivity). The content of stoichiometric EuO is clearly enhanced compared to sample type (i). However, the EuO/ITO interface is still dominated by over-oxidized phases. Thus, the YSZ buffer layer hinders oxygen diffusion from the substrate but cannot completely prevent it.

Our second strategy to avoid oxygen diffusion from the ITO virtual substrate is to grow EuO directly on ITO using the flux ratio of $J_{\mathrm{Eu}} / J_{\mathrm{O}}=1$, but with the substrate kept at room temperature. Oxygen diffusion in oxides typically follows an Arrhenius law, and the oxygen mobility in ITO is negligible at room temperature. $\uparrow$

For sample type (iii), the RHEED pattern in Fig. 3(j) reveals diffraction rings that are indicative of a polycrystalline EuO film. The corresponding HAXPES spectra in Fig. 3(k) show a

T From the data in ref. 26 the diffusion coefficient was extrapolated to be $1.5 \times$ $10^{-17} \mathrm{~cm}^{2} \mathrm{~s}^{-1}$ at room temperature. 

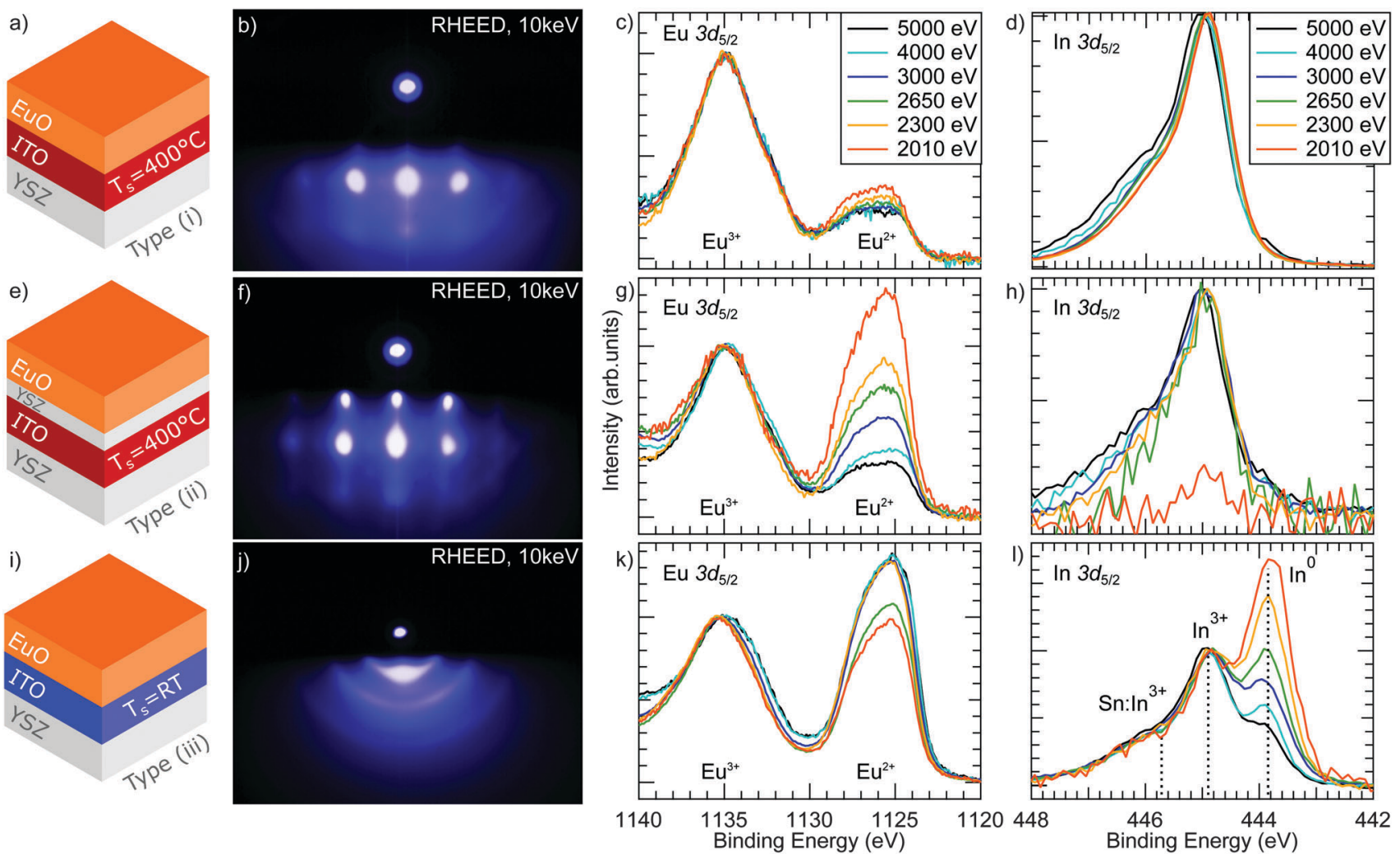

Fig. 3 Top row: sample type (i): $4 \mathrm{~nm}$ EuO grown at $400{ }^{\circ} \mathrm{C}$. Middle row: sample type (ii): $4 \mathrm{~nm}$ EuO grown at $400{ }^{\circ} \mathrm{C}$ with a $2 \mathrm{~nm}$ YSZ buffer layer. Bottom row: sample type (iii): $4 \mathrm{~nm}$ EuO grown at room temperature. (a, e and i) Schematic representation of the layer stacking. (b, $f$ and j) RHEED images taken with $10 \mathrm{keV}$. (c, g and k) HAXPES spectra of the Eu $3 d_{5 / 2}$ core level. (d, h and l) HAXPES spectra of the In $3 d_{5 / 2}$ core level. All spectra are normalized to the $\mathrm{Eu}^{3+}$ or $\mathrm{In}^{3+}$ components, respectively, except for the $2010 \mathrm{eV}$ spectrum in (h) which could not be normalized due to weak intensity.

$\mathrm{Eu}^{2+}$ ratio of about $60 \%$ and only small changes of the $\mathrm{Eu}^{2+} 3 \mathrm{~d}$ spectral intensity within the HAXPES depth profile. The relatively small variations of the $\mathrm{Eu}^{2+}$ intensity indicate that the chemical composition of the EuO layer is homogeneous.

Finally, we performed a complementing HAXPES depth profiling analysis of the In $3 \mathrm{~d}$ core levels of the buried ITO virtual substrate. The spectra of sample types (i) and (ii) are displayed in Fig. 3(d) and (h). They consist of two components: a sharp component located at $444.9 \mathrm{eV}$, which denotes trivalent $\mathrm{In}^{3+}$ (as expected for $\left.\mathrm{Sn}: \mathrm{In}_{2} \mathrm{O}_{3}\right)^{27}$ and a second, broad component located at $445.7 \mathrm{eV}$ which can be attributed to screening effects caused by the Sn dopants. ${ }^{28}$

In contrast, sample type (iii) reveals an additional spectral feature in the In $3 \mathrm{~d}$ core level as depicted in Fig. 3(1). For the largest photon energy (largest bulk sensitivity), the spectra are comparable to those of sample types (i) and (ii), but possess a small shoulder at the low binding energy side of the $\mathrm{In}^{3+}$ peak. This feature located at $443.9 \mathrm{eV}$ is characteristic for metallic $\mathrm{In}^{0} .^{27}$ The relative intensities $I_{\text {IтO }}$ and $I_{\text {In }}$ are shown in Fig. 4(b). $I_{\text {In }}$ increases with decreasing photon energy and dominates the spectrum at the lowest photon energies, i.e. at the highest surface sensitivity. Thus, we clearly find In metal present at the EuO/ITO interface of sample type (iii).

$\| I_{\text {ITO }}$ takes both ITO components i.e. $\operatorname{In}^{3+}$ and $\mathrm{Sn}: \mathrm{In}^{3+}$ into account (see ESI $\dagger$ ).
In order to estimate the thickness $d$ of the $\mathrm{In}^{0}$ layer from our HAXPES data we assumed the layer to be discrete. In this case one can apply the relation ${ }^{29}$

$$
\ln \left(1+\frac{R}{R^{\infty}}\right)=\frac{d}{\lambda_{\mathrm{In}}} \quad \text { with } R=\frac{I_{\mathrm{In}}}{I_{\mathrm{ITO}}} ; \quad R^{\infty}=\frac{I_{\mathrm{In}}^{\infty}}{I_{\mathrm{ITO}}^{\infty}}=\frac{\sigma_{\mathrm{In}} \lambda_{\mathrm{In}}}{\sigma_{\mathrm{ITO}} \lambda_{\mathrm{ITO}}}
$$

where $R$ is the ratio of the spectral intensities $I_{\text {In }}$ to $I_{\text {IтO }}$ as they are shown in Fig. 4(b), and $R^{\infty}$ is the ratio of intensities of bulk reference samples of these materials. The latter can be calculated from $\lambda$, i.e. the respective effective attenuation length (EAL)** and $\sigma$, i.e. the atomic density. As depicted in Fig. $4(\mathrm{~d})$, plotting $\ln \left(1+R / R^{\infty}\right)$ vs. $1 / \lambda_{\text {In }}$ gives a straight line that validates the assumption of a discrete $\operatorname{In}^{0}$ layer with sharp interfaces and yields a layer thickness $d_{\mathrm{In}}=(2.0 \pm 0.1) \mathrm{nm}$. The thermodynamic origin of the In layer will be discussed in Section 4.4.

In the same way, we analyzed the effective thickness of the EuO layer in samples (i) and (ii), see Fig. 4(c). Here, we assume that a discrete $\mathrm{EuO}$ layer is present on top of an $\mathrm{Eu}_{2} \mathrm{O}_{3}$ layer which is in contact with the substrate. For sample (ii), this approach yields a thickness of $d_{\mathrm{EuO}}=(1.3 \pm 0.1) \mathrm{nm}$. This is

** EALs are calculated with formula (8) of ref. 30. The therefor necessary inelastic mean free path (IMFP) and transport mean free path (TMFP) were simulated in SESSA. ${ }^{31}$ 

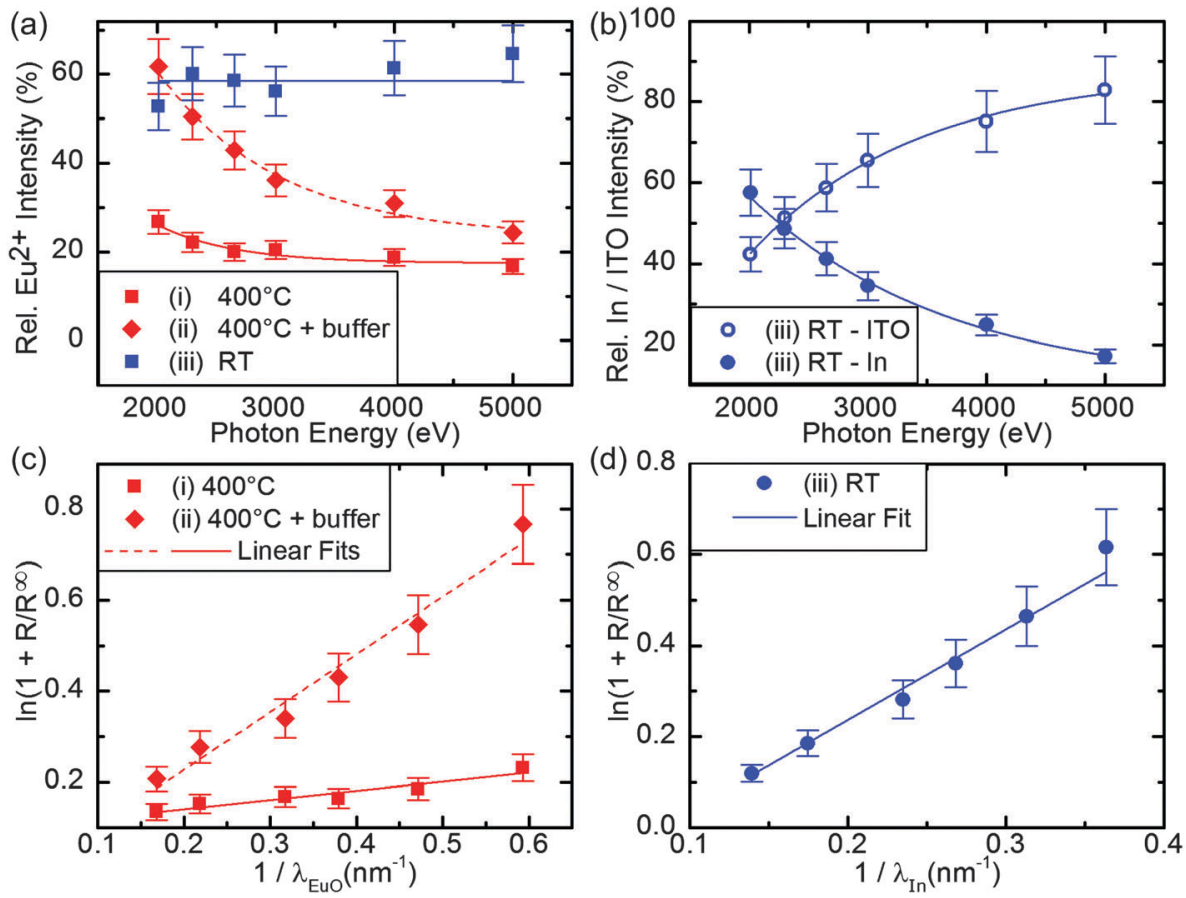

Fig. 4 (a) Relative $\mathrm{Eu}^{2+}$ intensity as estimated by fits to the HAXPES data shown in Fig. 3(c, g and k). (b) Relative intensity $I_{\text {ITO }}$ and $I_{\text {In }}$ of the HAXPES depth profile shown in Fig. $3\left(\right.$ (l). Lines in (a) and (b) are guides to the eye. (c) Determination of the EuO layer thickness for sample type (i) and (ii) gives $d_{\mathrm{EuO}}=(0.2 \pm 0.1) \mathrm{nm}$ and $d_{\text {EuO }}=(1.3 \pm 0.1) \mathrm{nm}$, respectively. (d) Determination of the In metal layer thickness for sample type (iii) gives $d_{\ln }=(2.0 \pm 0.1) \mathrm{nm}$.

about $1 / 3$ of the total film thickness which agrees reasonably well with the chemical composition of the film $\left(\right.$ ca. $\left.1 / 4 \mathrm{Eu}^{2+}\right)$. For sample (i), we find a value of $d_{\mathrm{EuO}}=(0.2 \pm 0.1) \mathrm{nm}$ which would be only $1 / 20$ of the film's thickness. This is much less than expected from the chemical analysis. We conclude that in the case of this sample we cannot assume that a discrete $\mathrm{EuO}$ layer is present but rather $\mathrm{EuO}$ islands on top of $\mathrm{Eu}_{2} \mathrm{O}_{3}$.

\subsection{Magnetic properties}

We complement the structural and chemical characterization of the EuO/ITO samples by probing their magnetic properties. Fig. 5(a) shows the respective $M(H)$ hysteresis loops recorded at $T=5 \mathrm{~K}$ and (b) shows $M(T)$ curves with an alignment field of 100 Oe.

As a reference, we show the magnetic properties of a thicker EuO film $(d=9 \mathrm{~nm})$ which was grown in the same way as sample (iii), i.e. deposition onto an ITO virtual substrate at room temperature. For this reference sample, we observe a square magnetic hysteresis loop with a coercive field of $H_{\mathrm{C}}=300$ Oe and a saturation magnetization of $6.5 \mu_{\mathrm{B}}$ per formula unit i.e. close to the bulk value of $7 \mu_{\mathrm{B}} /$ f.u. Furthermore, the $M(T)$ curve in Fig. 5(b) follows a Brillouin-like shape with a Curie temperature $T_{\mathrm{C}}=70 \mathrm{~K}$, thus, almost perfectly matching the bulk value of $69 \mathrm{~K}$. We conclude that EuO with bulk like magnetic properties can be obtained by room temperature deposition onto ITO virtual substrates.

For sample type (iii), we observe a hysteresis loop with $H_{\mathrm{C}}=320$ Oe and a saturation magnetization of $4.6 \mu_{\mathrm{B}}$ per formula unit. We note that the measured magnetization of all samples was normalized by the nominal film thickness of $4 \mathrm{~nm}$.
The effective EuO thickness, however, is much thinner due to the presence of $\mathrm{Eu}^{3+}$ phases which results in an underestimation of the saturation magnetization of the $\mathrm{Eu}^{2+}$ (i.e. EuO) phase. The corresponding $M(T)$ curve shows a Curie temperature of $T_{\mathrm{C}}=60 \mathrm{~K}$. This reduced Curie temperature is expected for EuO in the ultrathin film limit. ${ }^{34,35}$ Consistently, samples (i) and (ii) which contain more $\mathrm{Eu}^{3+}$ contaminants show magnetic hysteresis but with further reduced saturation magnetization. Also, $T_{\mathrm{C}}$ is reduced dramatically for these two samples due to a very low effective thickness of the EuO layer. The nominal layer thickness is already $4 \mathrm{~nm}$, but - as pointed out above - the effective thickness of the EuO layer is lower because these two samples contain mostly $\mathrm{Eu}_{2} \mathrm{O}_{3}$. Using eqn (1), we determined the thickness of the EuO layer in sample type (ii) to be $1.3 \mathrm{~nm}$.

The chemical composition of the samples as reflected by the spectral weight of $\mathrm{Eu}^{2+}$ or $\mathrm{Eu}^{3+}$ cations is, thus, fully consistent with their macroscopic magnetic properties.

\subsection{Thermodynamic analysis and discussion}

Combining the results on the three sample types, we derive the following picture of EuO synthesis on ITO: besides the oxygen gas which is supplied during the reactive MBE growth process, the ITO virtual substrate is an additional supplier of oxygen (Fig. 7(a)). The substrate is reduced by the presence of EuO to In metal while EuO over-oxidizes to $\mathrm{Eu}_{3} \mathrm{O}_{4}$ and $\mathrm{Eu}_{2} \mathrm{O}_{3}$. The chemical reactions taking place at the EuO/ITO interface are

$$
\begin{gathered}
6 \mathrm{EuO}+\frac{2}{3} \operatorname{In}_{2} \mathrm{O}_{3} \rightarrow 2 \mathrm{Eu}_{3} \mathrm{O}_{4}+\frac{4}{3} \mathrm{In} \\
4 \mathrm{Eu}_{3} \mathrm{O}_{4}+\frac{2}{3} \operatorname{In}_{2} \mathrm{O}_{3} \rightarrow 6 \mathrm{Eu}_{2} \mathrm{O}_{3}+\frac{4}{3} \mathrm{In}
\end{gathered}
$$



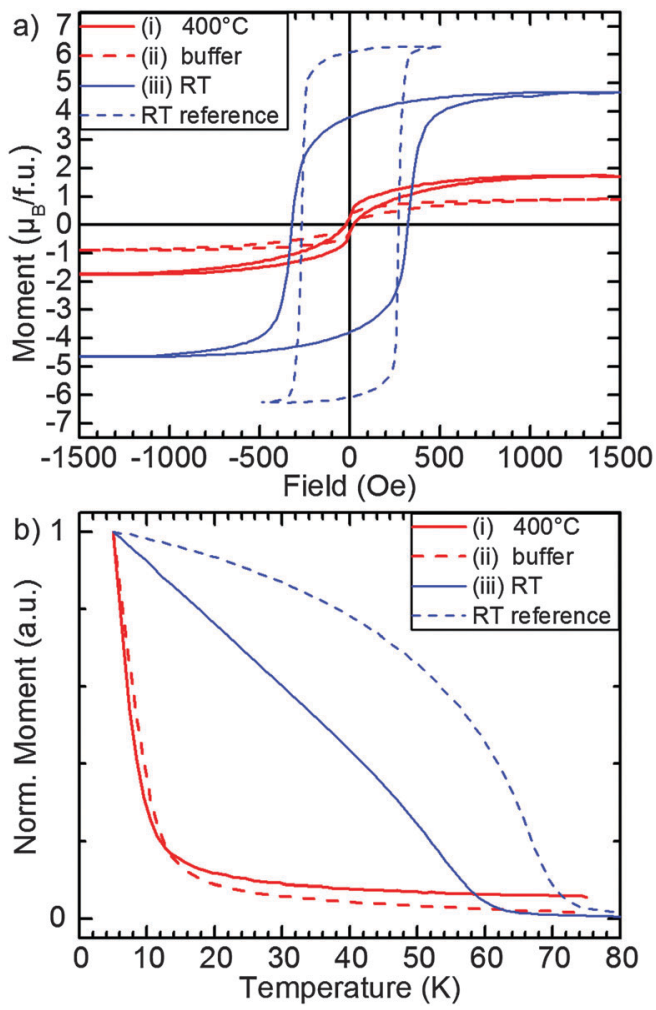

Fig. 5 Magnetic characterization of sample types (i)-(iii). For reference, a thicker film is shown (same growth procedure as sample type (iii) but with $d=9 \mathrm{~nm}$ ). (a) $M(H)$ hysteresis loops at $5 \mathrm{~K}$. (b) $M(T)$ curves with an alignment field of $100 \mathrm{Oe}$.

These reactions are thermodynamically favored as we derived from the Ellingham diagram ${ }^{36}$ shown in Fig. 6(a). Here, we compiled the change of the Gibbs free energy $\left(\Delta_{\mathrm{r}} G^{0}\right)$ as a function of temperature for any oxidation reaction of the metallic elements involved, which are

$$
2 \mathrm{Eu}+\mathrm{O}_{2} \rightleftarrows 2 \mathrm{EuO}
$$

$$
\begin{gathered}
6 \mathrm{EuO}+\mathrm{O}_{2} \rightleftarrows 2 \mathrm{Eu}_{3} \mathrm{O}_{4} \\
4 \mathrm{Eu}_{3} \mathrm{O}_{4}+\mathrm{O}_{2} \rightleftarrows 6 \mathrm{Eu}_{2} \mathrm{O}_{3} \\
\frac{4}{3} \mathrm{In}+\mathrm{O}_{2} \rightleftarrows \frac{2}{3} \mathrm{In}_{2} \mathrm{O}_{3} .
\end{gathered}
$$

$\Delta_{\mathrm{r}} G^{0}$ of reactions (2) and (3) can be easily estimated because

$$
\begin{aligned}
& \Delta_{\mathrm{r}} G^{0}(2)=\Delta_{\mathrm{r}} G^{0}(5)-\Delta_{\mathrm{r}} G^{0}(7) \\
& \Delta_{\mathrm{r}} G^{0}(3)=\Delta_{\mathrm{r}} G^{0}(6)-\Delta_{\mathrm{r}} G^{0}(7)
\end{aligned}
$$

Stable conditions for EuO and ITO are marked by the hatched areas in Fig. 5(a). We find that these areas do not overlap and that, consequently, $\Delta_{\mathrm{r}} G^{0}(2)$ and $\Delta_{\mathrm{r}} G^{0}(3)$ are negative (i.e. $<-300 \mathrm{~kJ} \mathrm{~mol}^{-1}$ $\mathrm{O}_{2}$ ) and thus both reactions are favored as indicated by the arrows in eqn (2) and (3).

Therefore, the given thermodynamic conditions prefer the formation of over-oxidized phases at a growth temperature $T_{\mathrm{S}}=400{ }^{\circ} \mathrm{C}$ and $J_{\mathrm{Eu}} / J_{\mathrm{O}}=1$ (sample type (i)). We note, that even with a flux ratio $J_{\mathrm{Eu}} / J_{\mathrm{O}}=2$ the abundance of over-oxidized phases could not be reduced (see ESI $\dagger$ ). Any excess Eu reacts with $\mathrm{O}$ from the substrate and, therefore, cannot re-evaporate. Consequently, for EuO synthesis on ITO substrates one cannot benefit from the distillation method.

Hence, oxygen diffusion from the ITO virtual substrate to the EuO film needed to be reduced and we proceeded in two ways: the insertion of a YSZ buffer layer (sample type (ii)) and the lowering of the growth temperature (sample type (iii)).

EuO is thermodynamically stable in contact with the YSZ buffer as it can be derived from the Ellingham diagram in Fig. 6(b). In absence of thermodynamic data for this particular YSZ compound (composition $\mathrm{Zr}_{0.87} \mathrm{Y}_{0.13} \mathrm{O}_{1.935}$ ), a reasonable approximation is examining the individual components (in particular $\mathrm{Zr}$ ). We thus compiled the change of the Gibbs free energy $\left(\Delta_{\mathrm{r}} G^{0}\right)$ for oxidation reactions of $\mathrm{Y}$ and $\mathrm{Zr}$ :

$$
\begin{aligned}
\frac{4}{3} \mathrm{Y}+\mathrm{O}_{2} & \rightleftarrows \frac{2}{3} \mathrm{Y}_{2} \mathrm{O}_{3} \\
\mathrm{Zr}+\mathrm{O}_{2} & \rightleftarrows \mathrm{ZrO}_{2} .
\end{aligned}
$$
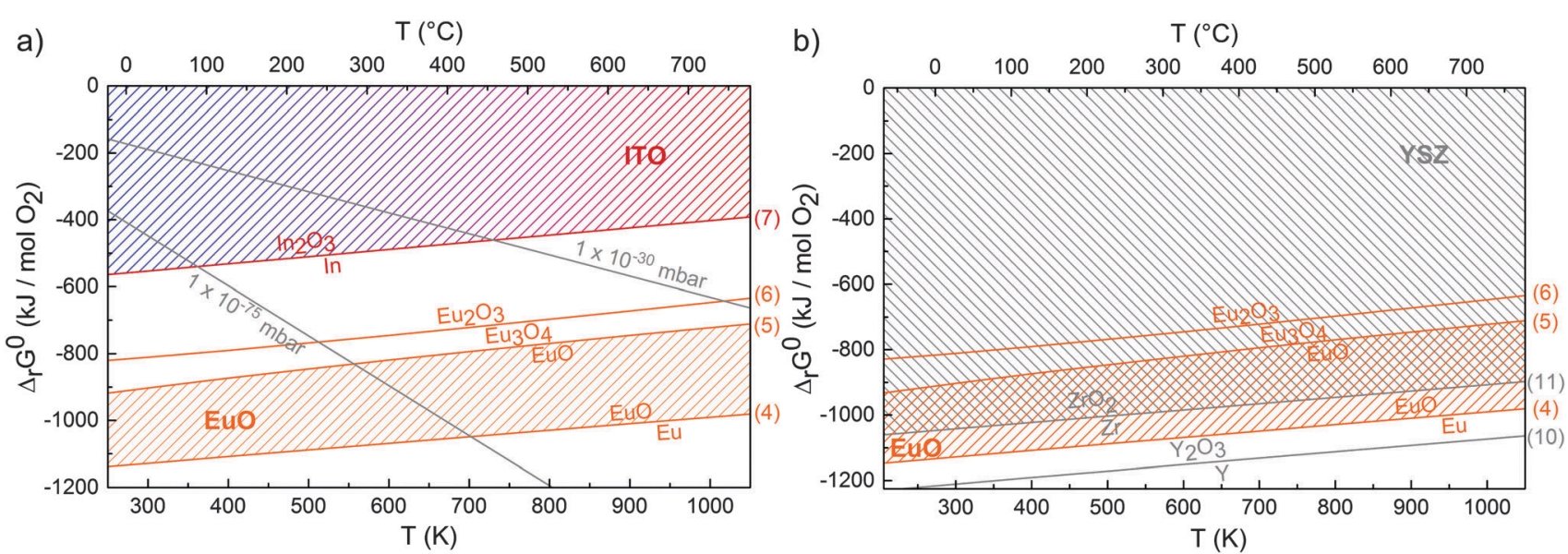

Fig. 6 (a) Ellingham diagram for oxygen reactions at the EuO-ITO interface calculated with thermodynamical data from ref. 32 and 33 . Gibbs free energy change of reactions (4)-(7) are indicated by straight lines. Stable conditions for EuO and ITO are marked by the hatched areas. Gray lines show constant $\mathrm{O}_{2}$ pressures. (b) Ellingham diagram for oxygen reactions at the EuO-YSZ interface. 
a) Type (i)

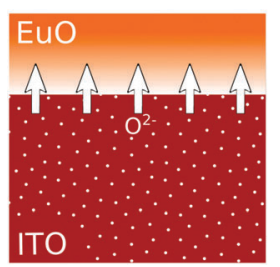

c) Type (iii)

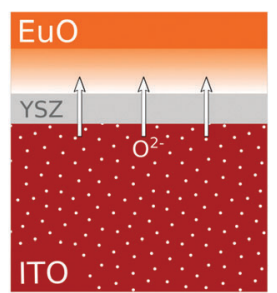

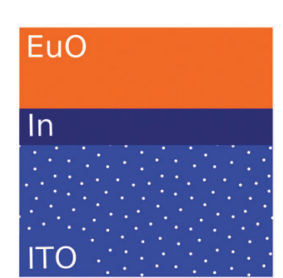

Fig. 7 Mechanisms of oxygen diffusion at the EuO/ITO-interface. (a) Type (i): $\mathrm{O}^{2-}$ from ITO diffuses to the EuO layer and acts as additional source of oxygen during growth. Oxygen is mobile at $400{ }^{\circ} \mathrm{C}$ so that oxygen vacancies at the ITO interface are replenished. (b) Type (ii): diffusion of oxygen to the EuO layer is reduced by an YSZ buffer layer. (c) Type (iii): $\mathrm{O}^{2-}$ reacts with the EuO layer at the interface but vacancies are not replenished so that the ITO interface suffers oxygen depletion and metallic $\mathrm{In}$ is formed.

Once more, stable conditions for EuO and YSZ are marked by the hatched areas in Fig. 6(b). In contrast to Fig. 6(a), these areas largely overlap signalling thermodynamic stability. HAXPES data of the $\mathrm{Zr} 3 \mathrm{~d}$ core level (see ESI $\dagger$ ) shows only $\mathrm{Zr}^{4+}$, which is the expected valence in YSZ, so that we can conclude that the buffer layer is homogeneous and, indeed, chemically stable.

Recalling the results on sample type (ii), the $2 \mathrm{~nm}$ thick YSZ buffer layer clearly improved the EuO stoichiometry. Thus, the buffer layer acts as a kinetic barrier that reduces oxygen diffusion. However, over-oxidized phases are still present at the EuO-YSZ interface. This means that the buffer layer is not a perfect diffusion barrier which is presumably due to the oxygen permeability of YSZ $^{37,38}$ (Fig. 7(b)).

Lowering the growth temperature reduces oxygen diffusion more effectively. A homogeneous EuO film containing 60\% stoichiometric EuO is obtained (sample type (iii)) but, in turn, the chemical composition of the ITO virtual substrate is substantially modified. After EuO synthesis, In metal is detected at the EuO/ITO interface (Fig. 7(c)). The observed reduction of ITO to In metal can solely be explained by the thermodynamic instability of ITO in contact with EuO as derived from Fig. 6(a).

As a last point, we address the fact that the reduction of ITO to In metal occurs only for sample type (iii), but not for types (i) and (ii). In the latter cases, a surplus of oxygen is present above the interface, but no oxygen deficiency is observed in the ITO substrate. We conclude, that oxygen vacancies occur at the surface of the ITO virtual substrate, but diffuse by thermal activation and are, thus, diluted below a measurable concentration. In the case of sample type (iii), the ITO virtual substrate suffers a significant oxygen depletion at the interface. Due to the reduced oxygen mobility at room temperature, these vacancies are not replenished and metallic In is present at the interface.

\section{Conclusions}

In summary, our study successfully combined a fundamental thermodynamical analysis in terms of solid state chemistry with HAXPES as a modern spectroscopy tool. Applying the nondestructive HAXPES technique yields a deep understanding of the chemical processes at the interfaces of the prototypical all-oxide hybrid EuO/ITO.

We have shown that ITO virtual substrates act as thermally activated, additional source of oxygen during reactive MBE growth of ferromagnetic EuO. At elevated growth temperatures over-oxidized phases with inferior magnetic properties were formed. Accordingly, the EuO growth was optimized by lowering the growth temperature. If synthesized at room temperature, EuO films show significantly improved chemical quality with sizeable magnetic properties.

Our thermodynamic analysis of oxygen reactions at the EuO-ITO-interface supports the presented HAXPES measurements. It is therefore promising to apply this approach to other oxide-oxide interfaces, in particular to calculate $\Delta_{\mathrm{r}} G^{0}$ accordingly for all oxygen reactions of the metals $M$ involved:

$$
\frac{2 x}{y} \mathrm{M}+\mathrm{O}_{2} \rightleftarrows \frac{2}{y} \mathrm{M}_{x} \mathrm{O}_{y}
$$

Suitable substrate and buffer layer materials are promising if they are thermodynamically stable in contact with the functional oxide overlayer. Even though MBE growth is largely governed by kinetic factors, thermodynamic stability signals the absence of a driving force for interface reactions.

Moreover, the oxygen conductivity turns out to be a major criterion for carefully choosing materials and synthesis temperatures. Generally, our methodical approach may serve as guideline for optimizing the growth and the interface properties of all-oxide heterostructures.

\section{Acknowledgements}

We acknowledge S. Döring for experimental support as well as O. Petracic and the Jülich Centre for Neutronscience for providing measurement time at the SQUID magnetometer. MM acknowledges financial support by HGF under contract No. VH-NG-811.

\section{References}

1 M. Müller, G.-X. Miao and J. S. Moodera, Europhys. Lett., 2009, 88, 47006.

2 G.-X. Miao, M. Müller and J. S. Moodera, Phys. Rev. Lett., 2009, 102, 076601.

3 R. B. H. Tahar, T. Ban, Y. Ohya and Y. Takahashi, J. Appl. Phys., 1998, 83, 2631-2645.

4 M. Matsubara, A. Schmehl, J. Mannhart, D. G. Schlom and M. Fiebig, Phys. Rev. B, 2012, 86, 195127.

5 Epitaxial Growth of Complex Metal Oxides, ed. G. Koster, M. Huijben and G. Rijnders, Woodhead Publishing, 1st edn, 2015.

6 P. G. Steeneken, PhD thesis, Rijksuniversiteit Groningen, Groningen, Netherlands, 2002.

7 R. W. Ulbricht, A. Schmehl, T. Heeg, J. Schubert and D. G. Schlom, Appl. Phys. Lett., 2008, 93, 102105.

8 R. Sutarto, S. G. Altendorf, B. Coloru, M. Moretti Sala, T. Haupricht, C. F. Chang, Z. Hu, C. Schüßler-Langeheine, 
N. Hollmann, H. Kierspel, H. H. Hsieh, H.-J. Lin, C. T. Chen and L. H. Tjeng, Phys. Rev. B, 2009, 79, 205318.

9 D. F. Förster, J. Klinkhammer, C. Busse, S. G. Altendorf, T. Michely, Z. Hu, Y.-Y. Chin, L. H. Tjeng, J. Coraux and D. Bourgault, Phys. Rev. B, 2011, 83, 045424.

10 C. Caspers, M. Müller, A. X. Gray, A. M. Kaiser, A. Gloskovskii, C. S. Fadley, W. Drube and C. M. Schneider, Phys. Rev. B, 2011, 84, 205217.

11 C. Caspers, A. Gloskovskij, W. Drube, C. M. Schneider and M. Müller, Phys. Rev. B, 2013, 88, 245302.

12 C. Caspers, S. Flade, M. Gorgoi, A. Gloskovskii, W. Drube, C. M. Schneider and M. Müller, J. Appl. Phys., 2013, 113, $17 \mathrm{C} 505$.

13 A. Schmehl, V. Vaithyanathan, A. Herrnberger, S. Thiel, C. Richter, M. Liberati, T. Heeg, M. Röckerath, L. F. Kourkoutis, S. Mühlbauer, P. Böni, D. A. Muller, Y. Barash, J. Schubert, Y. Idzerda, J. Mannhart and D. G. Schlom, Nat. Mater., 2007, 6, 882-887.

14 D. V. Averyanov, Y. G. Sadofyev, A. M. Tokmachev, A. E. Primenko, I. A. Likhachev and V. G. Storchak, ACS Appl. Mater. Interfaces, 2015, 7, 6146-6152.

15 K. J. Hubbard and D. G. Schlom, J. Mater. Res., 1996, 11, 2757-2776.

16 D. G. Schlom and J. H. Haeni, MRS Bull., 2002, 27, 198-204.

17 L. P. H. Jeurgens, W. G. Sloof, F. D. Tichelaar and E. J. Mittemeijer, Phys. Rev. B, 2000, 62, 4707-4719.

18 T. Mairoser, J. A. Mundy, A. Melville, D. Hodash, P. Cueva, R. Held, A. Glavic, J. Schubert, D. A. Muller, D. G. Schlom and A. Schmehl, Nat. Commun., 2015, 6, 7716.

19 C. Fadley, J. Electron Spectrosc. Relat. Phenom., 2010, 178, $2-32$.

20 J. Zegenhagen, Eur. Phys. J.: Appl. Phys., 2015, 70, 20701.

21 H. Ohta, M. Orita, M. Hirano, H. Tanji, H. Kawazoe and H. Hosono, Appl. Phys. Lett., 2000, 76, 2740-2742.

22 C. Buchal, L. Beckers, A. Eckau, J. Schubert and W. Zander, Mater. Sci. Eng., B, 1998, 56, 234-238.
23 C. Caspers, PhD thesis, Universität Duisburg-Essen, Duisburg, Germany, 2013.

24 M. Gorgoi, S. Svensson, F. Schäfers, G. Öhrwall, M. Mertin, P. Bressler, O. Karis, H. Siegbahn, A. Sandell, H. Rensmo, W. Doherty, C. Jung, W. Braun and W. Eberhardt, Nucl. Instrum. Methods Phys. Res., Sect. A, 2009, 601, 48-53.

25 E.-J. Cho, S.-J. Oh, S. Imada, S. Suga, T. Suzuki and T. Kasuya, Phys. Rev. B, 1995, 51, 10146-10149.

26 M. Quaas, H. Steffen, R. Hippler and H. Wulff, Surf. Sci., 2003, 540, 337-342.

27 M. Burgener and A. Goldmann, Surf. Sci., 2003, 540, 89-96.

28 V. Christou, M. Etchells, O. Renault, P. J. Dobson, O. V. Salata, G. Beamson and R. G. Egdell, J. Appl. Phys., 2000, 88, 5180-5187.

29 J. F. Watts and J. Wolstenholme, An Introduction to Surface Analysis by XPS and AES, Wiley-VCH, 1st edn, 2003.

30 C. J. Powell and A. Jablonski, Nucl. Instrum. Methods Phys. Res., Sect. A, 2009, 601, 54-65.

31 NIST Database for the Simulation of Electron Spectra for Surface Analysis, Version 2.0, ed. W. Werner, W. Smekal and C. J. Powell, National Institute of Standards and Technology, 2014.

32 R. J. Konings, O. Beneš, A. Kovács, D. Manara, D. Sedmidubský, L. Gorokhov, V. S. Iorish, V. Yungman, E. Shenyavskaya and E. Osina, J. Phys. Chem. Ref. Data, 2014, 43, 013101.

33 W. M. Haynes, CRC Handbook of Chemistry and Physics, CRC press, 95th edn, 2013.

34 R. Schiller and W. Nolting, Phys. Rev. Lett., 2001, 86, 3847-3850.

35 M. Müller, G.-X. Miao and J. S. Moodera, J. Appl. Phys., 2009, 105, 07 C917.

36 H. J. T. Ellingham, J. Soc. Chem. Ind., London, 1944, 63, 125.

37 P. Manning, J. Sirman, R. D. Souza and J. Kilner, Solid State Ionics, 1997, 100, 1-10.

38 K. L. Ngai, J. Santamaria and C. Leon, Eur. Phys. J. B, 2013, 86, 1-10. 